\title{
Percepción y uso de la tecnología por estudiantes de décimo año en las lecciones de Educación Cívica
}

\author{
Perception and use of technology \\ by students of 10th year in Civics lessons
}

Eduardo González Ayala

Profesor de la Sede de Occidente

Universidad de Costa Rica

San Ramón, Costa Rica

edgonaster@gmail.com

Ivany Villalobos Carranza

Profesora del Liceo Maurilio Alvarado de Tilarán

Dirección Regional de Cañas

Guanacaste, Costa Rica

ivisvr@gmail.com

Recibido: 18-IV-2012 • Aceptado: 04-VII-2012 • Corregido: 20-XI-2012

\begin{abstract}
Resumen: El presente articulo analiza el uso que los estudiantes de décimo año del Colegio Experimental Bilingüe de Palmares hacen de la tecnología de la que disponen, así como el acceso a la misma y la percepción que tienen sobre el uso de las TIC en las lecciones de Educación Cívica. El estudio utiliza encuestas $y$ grupos focales para acercarse a la realidad del estudiantado respecto al uso y aprovechamiento de la tecnología, así como a su percepción del proceso educativo, en particular en Educación Cívica. Se concluye que las y los jóvenes hacen un uso intensivo de la tecnología, de acuerdo a sus posibilidades de acceso, a la vez que participan de manera directa en el uso de la tecnología para fines propios, aunque no necesariamente vinculadas con su formación académica. Además, se evidencia que la percepción que tienen sobre la Educación Cívica es buena, pero que esta mejora cuando se hace uso de las TIC como estrategias metodológicas, siempre y cuando dichos recursos tecnológicos sean bien aprovechados.
\end{abstract}

\section{Introducción}

En una investigación de tesis de grado defendida en el año 2009, los autores expusieron resultados relacionados con la implementación de las Tecnologías de la Información y la Comunicación (TIC), por parte de docentes de décimo año en el Colegio Experimental Bilingüe de Palmares, y explicaron cómo dichas estrategias metodológicas eran valoradas y asimiladas por el alumnado. El presente artículo pretende rescatar dichos resultados, en particular lo concerniente a la percepción y las reacciones de las y los jóvenes respecto a aquellas lecciones de Educación Cívica en las cuales sus docentes utilizaron 
Palabras clave: Tecnología digital, Internet, Educación Cívica, educación.

\begin{abstract}
This article analyzes the use the students from the Bilingual Experimental School in Palmares make of the technology available, as well as the access to it and the perception on the use of ICT in Civic Education. The study uses surveys and focus groups to approach the reality of the students regarding their use and exploitation of technology, and their perception of the educational process, particularly in Civic Education. The study concludes that young people make heavy use of technology, according to their access possibilities, and that they participate directly in the use of technology for their own purposes, although not necessarily related to their academic training. Furthermore, it shows that the perception of Civic Education is good, but that this perception is improved when making use of ICTs as methodological strategies, provided such technological resources are well used.
\end{abstract}

Keywords: Digital Technology, Internet, Civic Education, education. herramientas tecnológicas y el impacto que dicho uso evidenció, así como las posibilidades de aprendizaje que propició.

El objetivo de la investigación fue vislumbrar la percepción, aceptación y motivación por parte de las y los estudiantes hacia la tecnología en las lecciones de Educación Cívica. A nivel metodológico, se realizaron encuestas al alumnado que cursaba décimo año, de las cuales se aprovechó la información para obtener un panorama sobre cómo se utilizaban las tecnologías en la clase y en qué circunstancias las aplicaba el profesorado. Como complemento, y para profundizar en la percepción, se llevaron a cabo grupos focales, los cuales permitieron visualizar las reacciones y el nivel de motivación del estudiantado, así como sus opiniones y críticas respecto a las lecciones en que se aplicó la tecnología digital. Estos resultados fueron triangulados con la información emanada de las entrevistas realizadas al personal docente.

En el presente trabajo, la percepción se entiende como aquel conjunto de actitudes y valoraciones que realizan las y los educandos de décimo año respecto a la utilización que realiza el cuerpo docente de la tecnología digital en las lecciones de Educación Cívica. Esta percepción se aprecia de manera notoria en los grupos focales que fueron desarrollados. Por su parte, se entiende por tecnología digital aquellos implementos utilizados por el profesorado en sus lecciones, por ejemplo la computadora, Internet y el video proyector, así como las actividades que estas tecnologías permiten.

En relación con las condiciones del contexto en el que se desenvuelven los y las estudiantes, conviene tener presentes algunos factores que posibilitan su acercamiento a la tecnología digital. De acuerdo con Zamora (2000), el cantón de Palmares presenta ciertas condiciones particulares, como su proyección social, al contar con acceso a servicios e instituciones, entre ellos asistencia en salud, un centro de la Universidad Estatal a Distancia (UNED), 
una sede de la Etapa Básica de Música de la Universidad de Costa Rica (UCR), la Cooperativa de Productores de Caña (CoopeIndia), la Biblioteca Pública y el Club de Amigos, entre otros.

Es conveniente recalcar que Palmares es una comunidad organizada, lo cual se evidencia mediante el aporte de varias instancias, entre ellas la Asociación Cívica Palmareña, además de las organizaciones comunitarias y las Juntas de Educación. Como apunta Rodríguez (2007), el cantón cuenta con una rica tradición popular orientada hacia las celebraciones religiosas, que demuestran su legado histórico y cultural.

En este contexto, el Colegio Experimental Bilingüe de Palmares presenta características especiales, específicamente en cuanto al acceso a tecnología digital, lo cual hace que un análisis de la aplicación de la misma y sus alcances en el ámbito educativo sea válido como un estudio de caso, pues además de presentar elementos particulares, la institución posee condiciones que la asemejan a otros centros educativos nacionales, como el hecho de ser un colegio público, diurno y académico.

La selección de dicho centro educativo obedeció a las especificidades de la institución en cuanto al uso de la tecnología digital en los procesos de enseñanza y aprendizaje. Esto debido a que el colegio cuenta con recursos como la Sala de conferencias, laboratorios de cómputo, Internet inalámbrica, biblioteca equipada y videoteca, entre otros. Además, la existencia de una Sala de conferencias (aula virtual), única en la región a nivel de colegios, permite a estudiantes y docentes tener acceso a videoconferencias y aprovechar sus recursos en el desarrollo de las lecciones. De esta forma, el estudiantado en Palmares posee condiciones particulares en cuanto al acceso a la tecnología en sus hogares propiamente, así como en la comunidad y el colegio.

Cabe mencionar que la investigación adquirió un carácter mixto, que tendió a decantarse hacia un enfoque cualitativo, al valerse tanto de grupos focales como de encuestas, para acercarse tanto a la percepción del alumnado como al acceso a los implementos tecnológicos. De acuerdo con Stake (1999), el contrastar instrumentos cualitativos y cuantitativos permite la triangulación entre las fuentes, lo cual facilita la elaboración de explicaciones alternativas y no encasilladas en una única fuente de información.

\section{Tecnología digital y aprendizaje}

La sociedad actual privilegia el uso de la tecnología digital y el acceso a la información, los cuales se convierten en herramientas vitales para el desarrollo de las actividades productivas y también para el desenvolvimiento habitual de las personas en sus múltiples ocupaciones, esto principalmente porque las TIC han "invadido" la cotidianidad y, en muchos casos, la privacidad de los individuos. Las sociedades se encuentran marcadas por la tecnología y muestran interdependencia, lo cual según Castells (2001) ha generado un sistema interrelacionado de comunicación que funciona en tiempo real, el cual reproduce y vende no solamente productos materiales, sino palabras, sonidos e imágenes, que son reciclados y retocados para acomodarse a las exigencias y gustos locales.

Esta interconexión a escala planetaria es lo que Castells (2001) ha denominado sociedad red, donde el propio sistema capitalista ha sido reestructurado, dando prioridad al capital sobre el trabajo, reduciendo la injerencia de los estados nacionales y colocando en un sitial privilegiado el acceso a la información y al conocimiento, por lo que se habla asimismo de una sociedad de la información, en la cual las personas se convierten no solo en receptoras, sino también en productoras, y esto en gran medida gracias a los avances tecnológicos.

No obstante, la visión lineal de que la tecnología conduce al progreso o al mejoramiento de las sociedades ha sido criticada argumentando que el desarrollo 
de la tecnología no lleva necesariamente a la satisfacción de las necesidades primordiales de los seres humanos, tal y como lo exponen Gutiérrez y Levis (2000, p. 69): "las TIC en sí mismas, como toda otra tecnología, no poseen cualidades intrínsecas que conduzcan inevitablemente a una mejora de las condiciones de vida".

Por lo tanto, los avances tecnológicos aplicados en la escuela tampoco implican directamente un mejoramiento en el proceso educativo. Visto lo anterior, la integración de las TIC al currículo es un proceso complejo que trasciende la relación establecida por tradición entre tecnología y progreso. El ambiente escolar introduce resistencias, tanto del alumnado como del profesorado, hacia la implementación de las TIC; dichas barreras están sustentadas en limitaciones de tiempo, entrenamiento, recursos y apoyo, puesto que, en muchas ocasiones, las y los educadores no cuentan con una capacitación adecuada para la utilización de las nuevas tecnologías (Dede, 2000). Además, las y los estudiantes se encuentran cotidianamente en contacto con muchos instrumentos propios de la sociedad de la información.

La aplicación de la tecnología digital en el aula reviste particular importancia y su alcance se convierte en un factor neurálgico por considerar, puesto que la democratización del acceso a la tecnología digital no avanza al mismo ritmo que el desarrollo de la misma. Es por ello que se habla de un analfabetismo tecnológico, es decir, de importantes sectores de la población que no han tenido la oportunidad de tener un contacto directo con las TIC y desconocen su funcionamiento. Autores como Piscitelli (2006) reconocen que los "inmigrantes digitales" son aquellas personas que han debido adaptarse a la época de la tecnología digital, mientras que los "nativos digitales" han crecido utilizando esta tecnología desde niños y la han incorporado en su vida cotidiana, por lo que la manejan de forma integral e intuitiva.
Ello implica que ciertos grupos sociales ven restringida su capacidad de acceder a los medios tecnológicos, o bien se les dificulta su aprovechamiento una vez que acceden a ellos. De esta forma, se evidencia que la conexión de las sociedades a la red de la información no necesariamente se lleva a cabo de forma rápida o eficiente, y se necesitan estrategias institucionales y estatales que faciliten a las y los ciudadanos la interacción con los recursos tecnológicos (Pavlik, 1994).

Estas limitaciones en el uso de la tecnología digital pueden conllevar restricciones en las capacidades de aprendizaje de las personas, no porque la tecnología sea indispensable en ese sentido, sino porque facilita el acceso a la información y es capaz de potenciar la producción del conocimiento en una sociedad cada vez más influenciada por el papel que desempeñan la información y el conocimiento como ventajas cualitativas en ámbitos como la producción y el trabajo:

Las nuevas fronteras de la sociedad capitalista, el quedar dentro o fuera de la misma, no las constituyen ni la propiedad de los medios o la posesión del capital ni la fuerza de trabajo, por importantes que sean. Las establece el poder acceder o no al capital intelectual (Gimeno, 2001, p. 65).

La sociedad actual presenta una alta dependencia de la tecnología digital, que condiciona el acceso a la información y a la capacidad de producir conocimiento, puesto que "las máquinas se convirtieron en verdaderas extensiones parciales y casi directas del cerebro" (Gallego, 1998, p. 116), las cuales tienen peso en la cotidianidad y han permeado de forma considerable los procesos educativos y la manera en la que las personas aprenden, particularmente las y los jóvenes. No obstante, la tecnología digital por sí misma no es capaz de sustituir el proceso de enseñanza y aprendizaje, por lo que debe considerarse que el uso de la información mediante la tecnología digital puede permitir la construcción del conocimiento si es mediado por adecuadas estrategias de aprendizaje. 
De lo anterior se deduce que la relación entre la tecnología digital y la educación debe conducir a la reflexión, ya que las TIC engloban formas desiguales de comunicar y de acceder a la información, lo cual permite la circulación de ideas diversas y de códigos de comunicación alternativos, que usualmente no se divulgan por medio de la escuela. Es decir, el sistema educativo reproduce, en muchas ocasiones, planteamientos que pueden ser superados en cantidad y contenido por las herramientas que la tecnología digital aporta a la vida cotidiana; sin embargo, este acceso cada vez más amplio a la información requiere una interacción constante con el proceso educativo para ser procesada e interiorizada.

Asimismo, Negroponte (2000) plantea que el acceso a la información se constituye en un elemento sustancial para comprender la vinculación de las sociedades y de las personas al mundo de la tecnología, situación que se manifiesta en el ambiente laboral, escolar y en la vida cotidiana, ya que el aislamiento de los avances en materia tecnológica implica un rezago que puede afectar el desarrollo de los individuos y de las sociedades, pues limita sus posibilidades de uso y aprovechamiento, más aún cuando la información obtenida mediante las TIC trasciende el plano físico y se reproduce por nuevos medios como Internet.

En este contexto, Internet ocupa un lugar privilegiado como medio para el acceso a la información y como posible herramienta complementaria en el proceso educativo. Lo anterior evidencia cómo la tecnología ha permeado con fuerza la vida cotidiana de las personas, y el ámbito educativo no es la excepción. La complejidad que exige el abordaje de Internet, de acuerdo con Briggs y Burke (2002), se sustenta en que esta tiene su propia lógica, pues permite a las personas acceder a una gran cantidad de información y se caracteriza por presentar un crecimiento acelerado.

No obstante, Internet no debe pensarse únicamente como un espacio "democrático", pues su acceso depende de condiciones socioeconómicas y de realidades disímiles entre países y regiones. Esta desigualdad en el acceso a la red ha sido recalcada por autores como Amar (2005), quien categoriza a las personas que no acostumbran navegar en esta Red, sea por falta de interés, desconocimiento o por dificultades económicas, como parte del "cuarto mundo".

La disyuntiva radica en la necesidad de acceder a las tecnologías digitales por parte de las y los estudiantes, indistintamente de lo siguientes factores: situación socioeconómica, ubicación geográfica o programa educativo en el cual estén inmersos, entre otros. De esta forma, la llamada brecha digital acusa una denuncia a las asimetrías existentes en la sociedad, en cuanto a la capacidad de acceso y el manejo de las tecnologías digitales más recientes, reflejadas en el sistema educativo de acuerdo con las características particulares de las comunidades.

Sin embargo, una vez que se accede a la Red, es posible la interiorización de nuevas potencialidades y las personas jóvenes tienden a familiarizarse rápidamente con las innovaciones. De esta forma, si la mediación es adecuada, la introducción y aplicación de nuevas tecnologías puede facilitar el acceso al conocimiento e incrementar los niveles de preparación del estudiantado. Esto principalmente por medio de la educación, que debería constituir una pieza clave para el desarrollo armónico de la sociedad y, al mismo tiempo, un importante insumo para lograr igualdad de oportunidades entre los seres humanos, contrariamente a la función preservadora del status quo que ha mantenido el sistema educativo tradicionalmente.

El uso de la tecnología digital y sus posibles beneficios para todas las personas constituye uno de los principios básicos de esta perspectiva de educación y formación que alimenta la idea expuesta por Bautista (2004), quien señala que el uso de la tecnología digital en la enseñanza debe orientarse hacia la mejora en los procesos educativos y debe buscar precisamente la 
integración de dichas tecnologías al sistema educativo. Es decir, la problemática no se sustenta únicamente en el acceso a las nuevas tecnologías, sino en la aplicación de herramientas adecuadas para que las y los educandos obtengan beneficios para su proceso de aprendizaje.

Para ello, es imprescindible tener presentes una serie de factores del contexto y comprender la realidad, necesidades e intereses propios de las y los estudiantes en la actualidad y sus relaciones con la tecnología, principalmente en el entendido de que este conocimiento puede favorecer la comprensión de los procesos actuales de aprendizaje.

\section{Acceso a la tecnología}

Un aspecto de especial importancia para valorar la recepción de la tecnología digital por parte de las y los alumnos consistió en determinar los principales implementos tecnológicos que posee su núcleo familiar, para poder realizar valoraciones a partir de los datos y la cobertura. Para ello, resulta importante vincular los resultados de la investigación con las condiciones propias del cantón de Palmares, el nivel socioeconómico del alumnado y las propias condiciones de acceso a la tecnología digital que presenta el colegio, por lo cual los resultados obtenidos no deben generalizarse de manera simplista a otras regiones o colegios del país.

Para dilucidar aspectos relacionados con el acceso a la tecnología por parte de las y los educandos de décimo año, conviene visualizar la presencia de equipos tecnológicos en los hogares relacionados con las TIC; para tal efecto, la Figura 1 muestra el porcentaje de aquellos dispositivos con los cuales se cuenta en las casas.

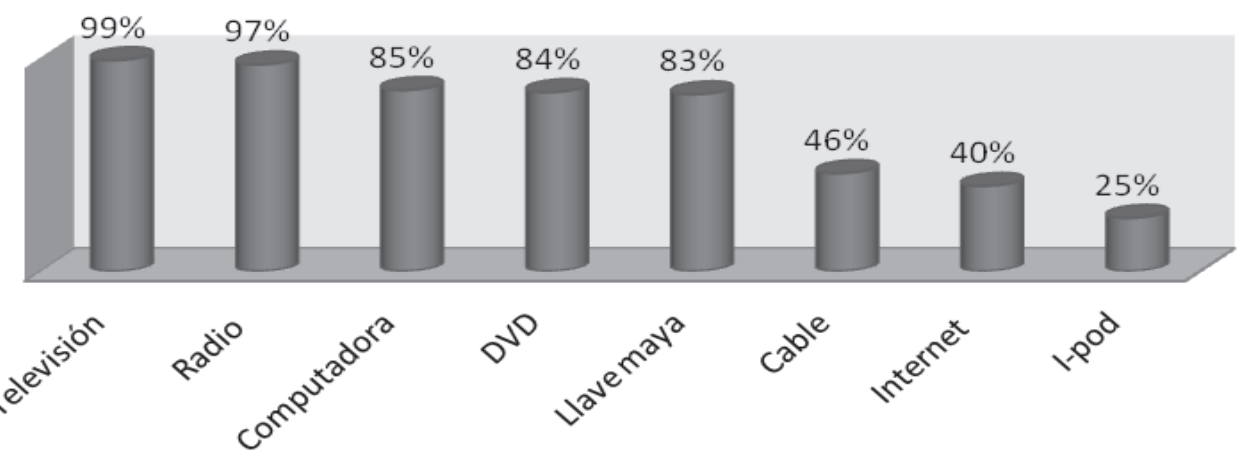

Figura 1. Recursos tecnológicos en el hogar de los estudiantes Fuente: Encuestas aplicadas a estudiantes, 2008.

La televisión y la radio son los recursos más comunes, lo que permite suponer que estos son los que con mayor frecuencia utilizan las y los jóvenes para entretenerse y aprovechar su tiempo libre, sobre todo la televisión, la cual está presente en el 99 por ciento de los hogares. Visto lo anterior, y observando lo planteado por Frabboni (1998), en la actualidad el papel del ocio trasciende al juego y la socialización, y pasa más por la recepción de imágenes y sonidos mediante implementos como el televisor; es 
decir, el tiempo libre se ha convertido en un objeto más de consumo.

Esta situación conlleva la necesidad de replantear el papel de la televisión y de los medios de difusión masiva como fuentes de entretenimiento o información en las sociedades contemporáneas:

Dada la omnipresencia de los medios de comunicación en la vida cotidiana (los españoles están por término medio 3 horas y 41 minutos ante la pantalla del televisor y el $65 \%$ de ellos tiene como única fuente de información medial la TV), se comprenderá la pertinencia de analizar la relación entre medios y enseñanza (Romano, 2003, p. 398).

Sobresale, entonces, la importancia de tener en cuenta el papel de los medios, particularmente de aquellos a los cuales el estudiantado tiene acces o constante, como elementos centrales en el proceso de educación informal y como insumos en la vida cotidiana que no deben ser subestimados por el sistema educativo formal. Por otra parte, recursos como la televisión por cable, Internet y los Ipods no están a disposición de todo el alumnado y solo cierto porcentaje (que no supera el 50 por ciento en estos 3 casos) tiene disponibilidad de poseerlos y utilizarlos en sus familias.

Es importante destacar también el uso de las computadoras en el hogar. En este sentido, las y los estudiantes manifiestan que el $85 \%$ poseen computadora en su casa. Este acceso demuestra la gran disponibilidad de estos aparatos en los hogares costarricenses en los últimos años. Sin embargo, no se pueden hacer extrapolaciones más allá del caso particular, pues según datos de la compañía Radiográfica Costarricense (RACSA), en el 2010 el 52 por ciento de los hogares en Costa Rica poseían computadora. Por ello, se deduce que el acceso de los hogares a los que pertenecen las y los educandos encuestados supera el promedio nacional, lo cual es un factor significativo a tomar en cuenta.

La alta disponibilidad de la computadora, al igual que la utilización de las memorias USB y los discos compactos, han transformado la manera en la cual el estudiantado busca la información para realizar sus trabajos del colegio, e inclusive, han variado la manera de adquirir, compartir y almacenar la información, dando lugar a la transformación de

...la capacidad de penetración de las nuevas tecnologías. Puesto que la información es una parte integral de toda actividad humana, todos los procesos de nuestra existencia individual y colectiva están directamente moldeados (aunque sin duda no determinados) por el nuevo medio tecnológico (Castells, 2001, p. 103).

Aunado a ello, las fuentes tradicionales para obtener información, como libros y periódicos, no han desaparecido pero han pasado a compartir su lugar de privilegio en relación con las TIC, principalmente por el uso de Internet y enciclopedias virtuales, que son las preferidas por los estudiantes.

De esta forma, el acceso a Internet ha aumentado considerablemente hasta alcanzar niveles importantes en los últimos años, sobre todo en América Latina. Según datos de RACSA correspondientes al 2010, el 53 por ciento de los habitantes en Costa Rica accedían a Internet. Estos datos reflejan un aumento en el uso de Internet y sitúan al país en una posición aceptable en cuanto a su cobertura. Aunado a ello, la radio y la televisión ocupan un papel importante como fuentes de información o difusores de conocimiento para la juventud, tendencia que igualmente se ha acentuado en los últimos años.

\section{Utilización de los recursos tecnológicos}

Es importante considerar de qué forma el alumnado asimila y se apropia del conocimiento relacionado con el manejo de las TIC, no solamente mediante la inducción que se le pueda ofrecer en el colegio, sino principalmente teniendo en cuenta sus propias experiencias personales, que evidencian su contacto con este tipo de 
tecnologías en ambientes como el hogar y la comunidad. El acceso a la tecnología posibilita ámbitos de aprendizaje que deben ser reconsiderados, a la vez que facilita "nuevas prácticas de lectura, escritura y sensibilidades" (Fainholc, 2004, p. 19).

No obstante, estos recursos son utilizados principalmente como medio de entretenimiento y pocas veces como insumo al proceso educativo. Inclusive, la computadora e Internet adquieren un papel significativo como formas de "pasar el tiempo", más que como complementos al proceso de aprendizaje, el cual tiende a considerarse por parte de las y los jóvenes como circunscrito a la institución educativa. Dicha realidad se evidencia en la Figura 2, donde se observan los criterios en torno a la utilización que de los dispositivos tecnológicos:

Cabe recalcar que el entretenimiento

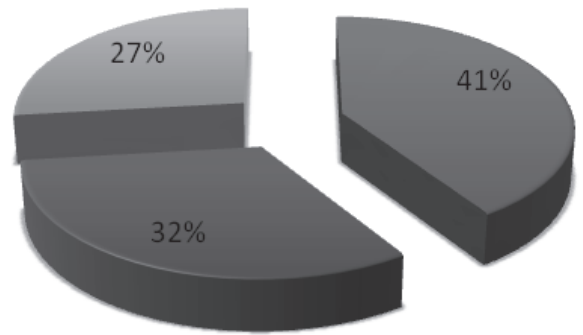

Entretenimiento

- Tareas o trabajos del colegio

$\square$ Obtener o trasladar información

Figura 2. Utilización de los recursos tecnológicos por parte de las y los estudiantes Fuente: Encuestas aplicadas a estudiantes, 2008.

es el fin principal de las y los jóvenes al recurrir a las TIC. En el caso del uso de la computadora e Internet, sirven principalmente para fines recreativos y de distracción, pues los utilizan para escuchar música, ver videos o películas, jugar videojuegos, visitar blogs, que son sitios en Internet donde es factible encontrar información, realizar preguntas y subir aportes en relación con un tema específico de interés para los usuarios. Además se utilizan para establecer relaciones con otras personas mediante sistemas de mensajería de texto y creación de perfiles en sitios como Facebook (Grupo focal sección 10-6, 19 de noviembre de 2008).

En estas páginas web el estudiantado se relaciona con un "grupo de amigos", algunos de ellos a quienes conocen en la realidad y otros personajes anónimos que se representan virtualmente mediante un perfil que los describe, en el cual se muestran sus datos personales, gustos, preferencias y aquellas personas con las cuales han entablado relación de "amistad virtual".

Mediante el intercambio de texto simultáneamente (chats), imágenes, videos y música, las y los alumnos establecen renovados estilos de comunicación que trascienden los intercambios personales e incluso los ámbitos relacionados con la tecnología digital, como el teléfono, la radio y la televisión. El ambiente de los videojuegos, que hasta hace algunos años se practicaba en solitario o bien con otros participantes al lado, actualmente, gracias a la Red, es posible jugar en un mismo escenario con personas de diferentes locaciones y países.

Este tipo de interacción virtual resulta significativa, pues establece una renovada área de construcción de identidades en la posmodernidad, donde las y los jóvenes se constituyen en un grupo etario 
particularmente susceptible de establecer vínculos de esta índole, gracias a Internet. No obstante, las identidades juveniles ya no pueden considerarse como inmutables o esenciales en la construcción de las identidades humanas, pues la sociedad actual favorece entornos -por ejemplo el mundo de las TIC- en los cuales estas identificaciones de las personas jóvenes se transforman en multicentradas, difusas, situacionales y transitorias:

La construcción de lo juvenil se da en contextos con mayor o menor grado de multiculturalidad y multilocación, de oportunidades enraizadas en condiciones económico-políticas con una distribución predominantemente dual. El mundo cibernético es parte de un cambio cultural que agudiza las diferencias y contribuye con nuevos códigos, condiciones y experiencias a los procesos de constitución identitaria de las juventudes (Krauskopf, 2007, p. 26).

Tales espacios de socialización virtual hacen que otras actividades, en las cuales usualmente el alumnado aprovechaba su tiempo libre, se vean disminuidas y colocadas en un segundo plano. Por lo tanto, áreas de esparcimiento supeditadas a la movilización (práctica de deportes, paseos, entre otros) se ven minusvaloradas en cuanto a sus posibilidades de atraer a la juventud, en tanto los espacios de entretenimiento virtual repuntan como actividades de ocio, que involucran mayores cuotas de individualización y sedentarismo, según lo sugieren los especialistas en medicina y psicología.

La revolución de los estilos de vida proporcionada por este tipo de entretenimiento tiene incidencia sobre la forma en la cual las y los estudiantes se visualizan a sí mismos, se relacionan y aprenden. Es decir, las investigaciones en educación en los tiempos actuales deben cuestionarse acerca no solamente de la didáctica, sino también colocar en el centro del debate el entorno en el cual se desarrolla el niño y el adolescente.

Por tanto, el uso de Internet como medio educativo adquiere una importancia medular, ya que ofrece insumos como el correo electrónico, chats, foros de discusión, videoconferencias y publicaciones, entre otros (Amador, 2004, p. 67). Sin embargo, el empleo que hacen las y los educandos se limita usualmente a la búsqueda de información específica de acuerdo con ciertos criterios establecidos previamente por las y los docentes, los cuales que no van más allá de la simple acumulación de texto sin mayor interpretación o análisis. Esto se constata al manifestar las y los educandos que la búsqueda de información en Internet, y su posterior transcripción, los aburre: “...uno va y hace el trabajo, y mientras está bajando la información chatea, para no aburrirse" (Grupo focal sección 10-6, 19 de noviembre de 2008).

Ante la transcripción acrítica de información y la idea de que el uso de la tecnología digital implica por sí mismo un proceso de aprendizaje, es importante recalcar que el aprendizaje es una construcción social que requiere la interacción constante de las personas y que, al involucrar la tecnología digital, se hace necesario un esfuerzo curricular y didáctico para lograr un mayor aprovechamiento de los recursos. La educación debe encaminarse hacia la capacidad del estudiante para discernir, entre el cúmulo de información de la que dispone, aquellos elementos que le pueden ser relevantes para su aprendizaje integral.

Internet se posiciona, más que un lugar de investigación, como un espacio virtual de entretenimiento para la juventud, que dedica importantes espacios de su tiempo libre a interactuar con las TIC de acuerdo con sus propios intereses. Esta realidad debe considerarse seriamente al tener en cuenta las necesidades del estudiantado en la actualidad en relación con el constante flujo de información que recibe y que debe procesar. En cuanto a la frecuencia con que las y los educandos encuestados acceden a Internet, la Figura 3 muestra una tendencia evidente.

Se debe subrayar el hecho de que únicamente el 5 por ciento de las y los 
alumnos afirmó nunca haber utilizado Internet, mientras que el restante 94 por ciento accedían al menos una vez a la semana e inclusive con mayor frecuencia, como lo demuestra el 29 por ciento que lo hacía diariamente.

La posibilidad de usar Internet con tanta frecuencia está relacionada con el acceso. Esta afirmación se puede sustentar al llevar a cabo un cruce de variables entre la disponibilidad de Internet en el hogar, la constancia y la motivación en su manejo. Es decir, de las y los estudiantes que poseen acceso en sus casas, el 62 por ciento lo utiliza diariamente y el restante porcentaje hace uso de la Red varias veces a la semana.

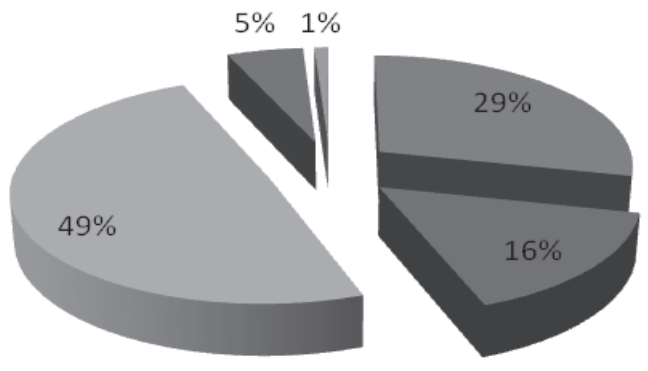

\author{
Diariamente \\ - Más de una vez a la \\ semana \\ Al menos una vez a la \\ semana \\ - Nunca \\ No responde
}

Figura 3. Frecuencia en el uso de Internet por parte de las y los estudiantes Fuente: Encuestas aplicadas a estudiantes, 2008.

Mientras tanto, las y los jóvenes que dependen de los Café Internet poseen una frecuencia de acceso más baja, ya que el 72 por ciento afirma utilizarlo al menos una vez a la semana y solamente un 13 por ciento accede diariamente por este medio. Esta limitación está relacionada principalmente con el factor económico, ya que en opinión de las y los colegiales el servicio de Internet, en aquellos comercios que lo brindan, es caro y en ocasiones puede salirse de su presupuesto en función de sus necesidades educativas.

A este respecto, el alumnado subraya la necesidad de acceso a la Red para cumplir con sus asignaciones en el ámbito académico y cómo el hecho de tener que pagar por el servicio significa para ellos un gasto extra: “...a veces uno ocupa bajar trabajos y está muy corto de dinero y tiene que ir al Internet, entonces que sea más en ese sentido, para no salir y bajar los trabajos aquí" (Grupo focal sección 10-6, 19 de noviembre de 2008).

La queja anterior del estudiantado refleja que la utilización de Internet los lleva a "bajar los trabajos", es decir, que no se dan procesos de aprendizaje significativos al elaborar sus propios proyectos, sino que dichas asignaciones son copiadas de otras que ya existen y en la mayoría de los casos el acopio de información se hace sin prestar mayor atención a la fuente.

Además, el alumnado reclama sobre la imposibilidad de acceder a Internet en el mismo colegio, ya que a pesar de contar con laboratorios de cómputo y el equipo necesario, estos se limitan a las clases de computación y 
a la modalidad de Bachillerato Internacional, en la cual algunos estudiantes de undécimo año, previamente seleccionados, se preparan para realizar una prueba de acreditación que tiene validez a nivel internacional, y para esta población estudiantil se reserva normalmente el uso de la Red.
Dadas las dificultades para utilizar el servicio en la institución, las y los educandos recurren a otros sitios, principalmente los Café Internet. Con el afán de aclarar lo expuesto anteriormente, la Figura 4 expresa los lugares en los cuales hacen uso de Internet:

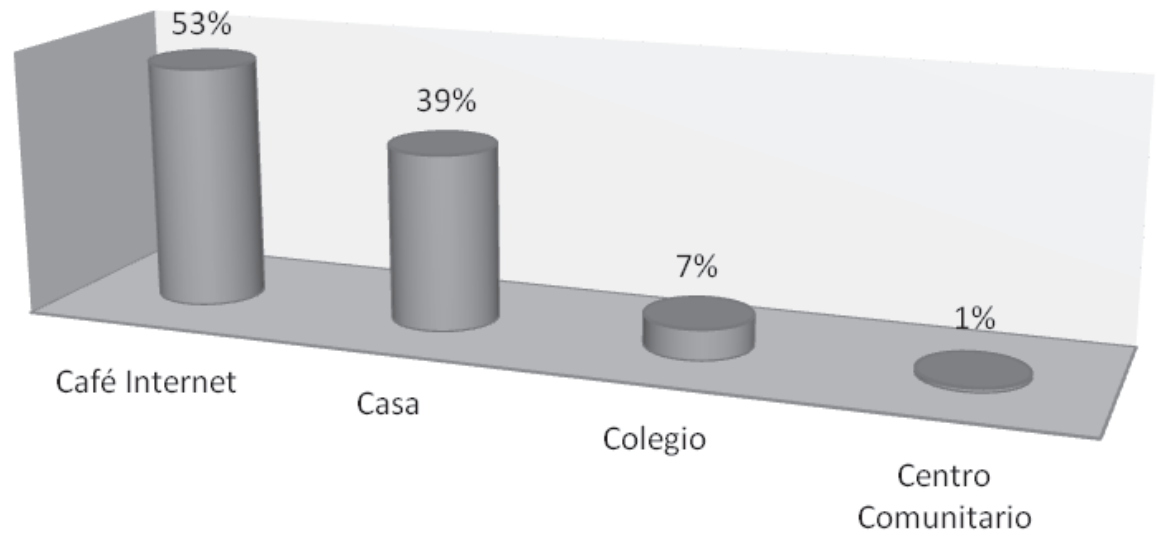

Figura 4. Lugares de uso de Internet por parte de las y los estudiantes Fuente: Encuestas aplicadas a estudiantes, 2008.

De esta forma, es notorio que las y los alumnos utilizan mayoritariamente los Café Internet (53 por ciento de las menciones), esto por cuanto la mayoría no posee el servicio en sus hogares. Solo el 39 por ciento tienen acceso a Internet en sus casas, pues cuentan con un entorno familiar con mayor estabilidad socioeconómica y por ello gozan de facilidades para obtener y aprovechar los insumos tecnológicos.

No obstante, esta realidad contrasta con las y los estudiantes que deben acudir preferentemente a un Café Internet y con los que no utilizan regularmente la Red. Esta situación remite a considerar el contexto latinoamericano, y en particular, las posibilidades de acceso a Internet de la las personas jóvenes en otros contextos:

El riesgo, enorme y cierto, es que, si los sistemas multimedia se convierten en el principal vector del proceso educativo, se ahonde cada vez más en la brecha cultural y, por ende, la desigualdad de oportunidades, entre los niños y jóvenes del mundo rico -con fácil acceso a tecnología - y los hijos de las comunidades con menores recursos (Gutiérrez y Levis, 2000, p. 69).

De esta forma, un sector de la población corre el riesgo de quedar excluido de las posibilidades que ofrece el mundo virtual. Mientras tanto, aquellos jóvenes "conectados" aprovechan estos recursos tanto para buscar información relacionada con sus actividades académicas, como para ensanchar sus espacios de distracción. No obstante, las posibilidades de investigación que esta Red ofrece no son desarrolladas por el estudiantado, porque no poseen un acompañamiento que les permita explorar esas destrezas. Los trabajos del colegio se limitan a la búsqueda de información para luego pasarla al papel y por ello no despiertan un mayor interés. 
Respecto al acceso a los medios tecnológicos en el colegio, las y los jóvenes recalcan que este es limitado para ellas y ellos, pues deben pedirlos con mucho tiempo de antelación y aún así no es seguro su préstamo, ya que depende de la disponibilidad, y en ocasiones el equipo es solicitado por algún docente que lo requiere y se le da prioridad, al igual que al estudiantado de Bachillerato Internacional.

Es decir, la existencia de los equipos de que dispone la institución no garantiza una aplicación significativa de las TIC en el proceso de enseñanza y aprendizaje, ya que los mismos estudiantes se quejan de las difíciles condiciones para su acceso, sobre todo en el caso de la Sala de Conferencias e Internet:

Mientras que a los estudiantes se nos habilite la tecnología, que no los mantengan ahí encerrados, encajonados y sin uso, todo va a ser mejor, porque por ejemplo nos ponen a hacer una investigación, entonces yo tengo que ir al Internet, pagar por hacer la investigación, después tengo que ir a la casa a buscar una computadora, a uno se le hace mucho más difícil, en vez de tener todo aquí (Grupo focal sección 10-11, 13 de noviembre de 2008).

De esta forma, se recalca el hecho de que muchos equipos, principalmente aquellas computadoras destinadas en primera instancia al uso de Internet por parte de las y los educandos, así como la conexión inalámbrica, se encuentran subutilizados en la institución y son muy difíciles de acceder para el alumnado, que debe salir al exterior para optar por tales beneficios,

En este sentido, el servicio de Internet se limita a un reducido número de estudiantes y al profesorado, que posee mayor libertad en cuanto a su uso. La queja principal del alumnado enfatiza el hecho de que los recursos tecnológicos existentes en la institución (equipos, Internet, Sala de Conferencias), se encuentran limitados para su aprovechamiento, por lo cual deben aprender acerca de su utilización por su propia cuenta y no por una inducción llevada a cabo en el colegio.
También queda manifiesta la visión que las y los alumnos desarrollan respecto a un proyecto de investigación. Para ellas y ellos, investigar se limita a visitar un Café Internet y extraer de la Red la información necesaria, la cual es reproducida sin mayor análisis crítico en un documento presentado para obtener una calificación. De esta forma, se tergiversa el sentido de un proceso de investigación y se le reduce a la simple transferencia de información.

Respecto al uso de la Sala de Conferencias de que dispone la institución, el estudiantado sostiene que puede acceder a ella solamente si se solicita con mucho tiempo de anticipación, pues se utiliza prioritariamente para actividades como teleconferencias; el cuerpo docente se siente inhibido para manipularla a causa de la incertidumbre sobre la posibilidad de desarrollar una clase completa o cumplir con objetivos establecidos previamente.

\section{Percepción sobre el uso de la tecnología en el aula}

Las opiniones que el alumnado esboza sobre el uso de las TIC en el aula reflejan una serie de prácticas que limitan las posibilidades de aprendizaje y manifiestan la visión que poseen las y los profesores sobre el empleo de la tecnología. Las y los estudiantes esperan una mayor participación en el uso de estos recursos y un creciente protagonismo en cuanto a su aprovechamiento, ya que en la mayoría de los casos solo pueden acceder a espacios como el aula virtual. El cuerpo docente delega en el estudiantado la responsabilidad de organización de las actividades que allí se desarrollan, las cuales a causa de la improvisación, no van más allá de la presentación de videos y diapositivas, técnicas que se aplican igualmente en las aulas, y dado el limitado conocimiento e iniciativa que poseen las y los educadores cuando utilizan estas tecnologías digitales, son las y los alumnos quienes deben guiar el desarrollo de la clase en dichas situaciones. 
Esta realidad es significativa, pues no refleja una aceptación total de aquellas lecciones en las cuales se utiliza la tecnología. Más aún, el alumnado se muestra crítico respecto a la forma en la cual sus docentes hacen uso de videos y presentaciones, muchas veces descontextualizados y sin un apoyo metodológico que permita vincular estas técnicas con conocimientos previos y con el desarrollo de nuevas habilidades, orientadas al discernimiento en torno a la escogencia de la información que es pertinente. Es decir, tal y como lo plantea Gimeno (2005), el solo acceso a la Red no garantiza la interiorización de conocimientos y destrezas.

Aunado a lo anterior, el uso de las TIC por parte del cuerpo docente adolece de una serie de carencias relacionadas con su capacitación y su disponibilidad para innovar, pues queda en evidencia cómo no se utilizan los equipos por desconocimiento o simple comodidad:

A veces si tienen recursos pero no los prestan siempre, o los profesores no los usan, entonces eso es lo que puede afectar; a veces si hay, un tele o un DVD, pero el profesor no lo usa, desarrolla la clase de la manera más fácil para él y no de la manera más fácil para aprender uno (Grupo focal sección 10-6, 19 de noviembre de 2008).

En cuanto a la utilización de la tecnología digital en las clases, las y los estudiantes consideran que no se realiza una adecuada aplicación, pues en muchas ocasiones su uso se limita a la proyección de un video o de una presentación en Power Point, pero no va más allá. Opinan además que la utilización de las TIC debería profundizarse, tanto en las clases de Estudios Sociales como las de Educación Cívica, adaptando de una mejor manera el uso de los implementos tecnológicos a los contenidos de dichas asignaturas.

El estudiantado coincide en que espera recibir lecciones más interactivas, en las cuales no solamente se utilice la tecnología digital, sino que ellas y ellos puedan participar activamente en el desarrollo de las actividades. Aquellas lecciones magistrales en las cuales predomina la comunicación verbal unidireccional, o en las que se utilizan técnicas tradicionales como el empleo de libros y fotocopias, son calificadas como cansadas y tediosas.

Estas técnicas se consideran además monótonas, pues al ser empleadas por las y los docentes constantemente, se convierten en reiterativas, lo que provoca cansancio y apatía en el alumnado. Igual ocurre con las temáticas que se desarrollan en clase, sobre todo en Educación Cívica; en esta materia, las y los educandos insisten en la necesidad de incorporar nuevos temas, a la vez que se revisen los ya existentes y se muestren de una forma más atractiva, porque de la manera en que se aborda actualmente:

Se hace demasiado repetitivo y tanta represión, uno se vuelve loco, se cansa, y todos los años es lo mismo: "ya vamos para Cívica, vamos a ver la misma materia de siempre"; de sétimo hasta décimo casi hemos visto lo mismo, y yo para Cívica casi nunca estudio, uno ya lo sabe, se torna en algo más personal y no tanto de materia (Grupo focal sección 10-11, 13 de noviembre de 2008).

En este sentido, la utilización de videos en el aprendizaje es una idea apoyada por el estudiantado, al sostener que el estímulo visual es un insumo muy importante para su aprendizaje, así como un complemento y un refuerzo a la materia vista mediante la aplicación de metodologías tradicionales. Se insiste en que dicho recurso debe ir acompañado de la explicación de las y los profesores o de algún método escrito que permita complementar lo visto en el video.

Sin embargo, las y los jóvenes acentúan el hecho de que el profesorado prefiere llevar a cabo sus clases de la forma que represente el mínimo esfuerzo para ellos y en muchas ocasiones no utilizan la tecnología porque “...no saben cómo, o no lo preparan bien o no consiguen material, o simplemente les da pereza; no les gusta complicarse, van más a lo tradicional, con 
el libro y lo mismo de siempre" (Grupo focal sección 10-11, 13 de noviembre de 2008).

La posición del alumnado revela las deficiencias en cuanto al planeamiento $\mathrm{y}$ desarrollo de nuevas alternativas didácticas, por lo que las lecciones derivan en el empleo constante de exposiciones magistrales, libros y fotocopias, y cuando se utilizan los medios tecnológicos, su uso no refleja una suficiente preparación, sino que se considera una forma de "entretenimiento", más que un esfuerzo por plasmar metodologías integrales que busquen reforzar y aclarar contenidos y procesos de manera innovadora.

No obstante, la opinión de las y los estudiantes respecto al atractivo de las lecciones de Educación Cívica es favorable, ya que el 63 por ciento considera interesantes las clases. En estos resultados influyen la motivación y las condiciones propias del contexto en el cual se lleva a cabo el proceso educativo, así como las particularidades de la materia y las actitudes personales o del grupo de estudiantes. Ello implica además, visto desde otro punto de vista, que a más de un 30 por ciento de las y los alumnos no les parece atractiva la clase, lo cual resulta significativo en términos de mejoramiento de la calidad de la educación y de la necesidad de estrategias didácticas pertinentes.

Los datos contrastan con la idea tradicional de que el estudiantado muestra un total desapego hacia la Educación Cívica, por considerarla una asignatura poco agradable. La apatía no está relacionada con la materia en sí, sino más bien con la repetición constante de las temáticas en los diferentes niveles y con las deficiencias metodológicas que afectan a las lecciones, las cuales no permiten una un mayor impacto en el proceso de aprendizaje.

Dichos datos apuntan a que el gusto que puede desarrollar el alumnado hacia la clase de Educación Cívica tiene mucho que ver con la forma en la cual el cuerpo docente se desempeñe, participando como facilitador y diseñador de técnicas significativas y atractivas de aprendizaje. Al ligar lo anterior con las TIC, se debe considerar que

Las nuevas tecnologías están revolucionando el mundo y sus efectos en la educación, aunque todavía incipientes, deben analizarse con mucha seriedad a fin de incorporarlos adecuadamente. No se trata de sustituir al docente por una pantalla, ni el libro por una computadora, sino de utilizar estas nuevas herramientas para hacer más agradable y eficiente la enseñanza (Villasuso, 2005, p. 19).

En ese sentido, cabe recalcar que las y los alumnos consideran las lecciones de Educación Cívica en las que se aplican las TIC como más sugerentes y atractivas. Para visualizar de manera más acertada la relación existente entre la motivación y el uso de la tecnología, se presenta la Figura 5.

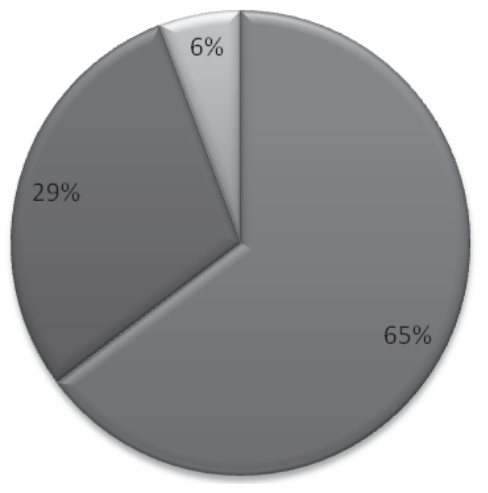

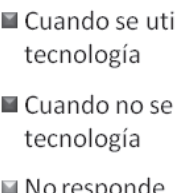

$\square$ No responde

Figura 5. Estudiantes consideran la clase atractiva

Fuente: Encuestas aplicadas a estudiantes, 2008. 


\section{Conclusiones}

En relación con el acercamiento de los jóvenes a la tecnología digital, se constató cómo el acceso a medios de entretenimiento, como la televisión, es prácticamente universal. Las computadoras se han expandido de una forma notable, llegando a la mayoría de los hogares de los jóvenes con los que se trabajó, y convirtiéndose en un instrumento que sirve tanto para el entretenimiento como para fines educativos, en el sentido de cumplir con las labores del colegio o administrar la información necesaria para el estudio.

Sin embargo, a pesar de la importancia que para los jóvenes tiene la Internet, solo el $40 \%$ de ellos tiene acceso en sus casas, por lo que los restantes estudiantes lo utilizan por otros medios. Sobre este punto, un elemento por considerar respecto a la disponibilidad de equipos en el colegio, reside en la limitación en el acceso para los estudiantes, sobre todo en el caso de Internet. Se cuenta con equipos de avanzada, pero no se encuentran a disposición de todos los jóvenes, la mayoría de los cuales deben salir de la institución y utilizar el servicio en los llamados "Café Internet", lo cual les implica un gasto económico importante.

A pesar de ello, Internet es el medio cuya expansión implica mayores posibilidades para los jóvenes. A través de las redes sociales, los blogs y otras páginas similares, ellos invierten su tiempo y aprovechan la Red para desarrollar nuevos intereses vitales que tienen que ver con las amistades, el deporte, la religión y el estudio. Más aún, Internet posibilita formas de comunicación innovadoras mediante los chats y el uso de las redes sociales, lo que permite el establecimiento de códigos entre ellos y nuevas formas de disponer del tiempo libre.

Respecto al uso de la Internet para fines educativos, se encuentra limitado al cumplimiento de las tareas propias del colegio y de proyectos de investigación que ter- minan reduciéndose a la búsqueda y transcripción de la información. La utilización que el estudiantado hace de este recurso con fines académicos en casas o comercios se circunscribe a la búsqueda de información, la cual luego se traslada e imprime tal y como fue encontrada en la red, lo cual significa que no existe mayor aporte o labor investigativa por parte de los jóvenes, pues la dinámica de los trabajos extra clase en el colegio no facilita el desarrollo de ideas propias y la capacidad de investigación.

En cuanto al interés que manifiesta el alumnado respecto a las lecciones de Educación Cívica, este no se encuentra supeditado a la utilización o no de las TIC por parte de los profesores; no obstante, la integración de las tecnologías digitales en el desarrollo de los contenidos sí mejora la impresión que tienen los jóvenes de la asignatura. En ese sentido, desempeña un papel medular la motivación que logre despertar el docente y la manera en que presente las lecciones, pues las estrategias interactivas evidencian una mayor atracción para los estudiantes. La presente investigación sugiere que la Educación Cívica no es necesariamente una materia que despierte aversión o apatía entre el estudiantado, como tradicionalmente se ha intentado presentar.

Un aspecto por recalcar es la percepción del alumnado respecto a la falta de planificación de las lecciones por parte de los docentes, donde se evidencia cómo las TIC son utilizadas como una forma de intentar presentar un recurso innovador por sí mismo, pero al utilizarlo con poca planificación, el impacto en el aprendizaje es limitado y ello hace que el estudiantado considere que los docentes no dedican el suficiente esfuerzo en la planificación de las clases o en la búsqueda de estrategias didácticas que faciliten el aprendizaje.

La responsabilidad institucional también está presente, pues los jóvenes en la actualidad se encuentran en contacto con la tecnología digital y hacen uso de ella como medio de comunicación y en sus activida- 
des cotidianas, por lo que demandan a su vez formas de aprendizaje en las cuales se integren la tecnología y los conocimientos que ellos han desarrollado, así como otras habilidades y destrezas que les sirvan en su vida futura, ya sea en el estudio o el trabajo.

Estas necesidades llevan a reflexionar sobre el proceso de enseñanza y aprendizaje formal en el ámbito institucional, el cual no debe desligarse de las actividades cotidianas que llevan a cabo los jóvenes, en las cuales utilizan de manera constante la tecnología. Teniendo en cuenta lo anterior, la población docente debe considerar este aprendizaje informal como un insumo y no olvidarlo en el desarrollo de las lecciones, como parece suceder con algunos ejemplos presentes en esta investigación. La utilización integral de la tecnología digital en las lecciones de Educación Cívica conlleva compromiso, responsabilidad en la planificación e ingenio para la innovación.

\section{Referencias}

Amador, L. (2004). Las Tecnologías de la Información y la Comunicación y la formación en entornos virtuales. Revista Complutense de Educación, 15 (1), 51-74.

Amar, V. (2005). Tecnología y educación: el resurgir del cuarto mundo. Historia Actual On Line. (8), 175-182. Recuperado de http:// www.historia-actual.com/HAO/ Volumes/Volume1/Issue8/eng/ v1i8c14.pdf

Bautista, A. (2004). Calidad de la educación en la sociedad de la información. Revista Complutense de Educación. 15 (9), 509-520.

Briggs, A., y Burke, P. (2002). De Guttenberg a Internet. Una historia social de los medios de comunicación. Madrid: Taurus.
Castells, M. (2001). La era de la información: Economía, sociedad y cultura. La sociedad Red (2da Ed.). Madrid: Alianza Editorial.

Dede, C. (2000). El proceso de incorporación progresiva de las innovaciones educativas basadas en la tecnología. En C. Dede (Comp.), Aprendiendo con tecnología. Buenos Aires: Paidós.

Fainholc, B. (2004). Lectura crítica en Internet. Análisis y utilización de los recursos tecnológicos en educación. Rosario: Homo Sapiens Ediciones.

Frabboni, F. (1998). El libro de la pedagogía y la didáctica: la educación. Madrid: Editorial Popular.

Gallego, R. (1998). Discurso constructivista sobre las tecnologías. Una mirada epistemológica. Bogotá: Cooperativa Editorial Magisterio.

Gimeno, J. (2001). Educar y convivir en la cultura global. Las exigencias de la ciudadanía. Madrid: Ediciones Morata.

Gutiérrez, M.L., y Levis, D. (2000). ¿Hacia la herramienta educativa universal? Enseñar y aprender en tiempos de Internet. Buenos Aires: Ediciones Ciccus - La Crujía.

Krauskopft, D. (2007). Dimensiones $y$ pertenencias en la construcción de identidades juveniles en Centroamérica. En M. Zimmerman y G. Baeza (Coords.), Estudios culturales centroamericanos en el nuevo milenio. Houston: LACASA.

Negroponte, N. (2000). El mundo digital. Un futuro que ya ha llegado. Barcelona: Ediciones B.

Pavlik, J. (1994). Citizen access, involment, and freedom of expression in an electronic envionment. En 
John Pavlik y Frederick Williams (Eds.), The people`s right to know: media, democracy and the information highway. New Jersey: Lawrence Erlbaum Associates Publishers.

Piscitelli, A. (2006). Nativos e inmigrantes digitales. ¿Brecha generacional, brecha cognitiva o ambas y más aún? Revista Mexicana de Investigación Educativa. 11 (28), 179-185.

Radiográfica Costarricense (RACSA). $53 \%$ de los habitantes acceden a internet. Recuperado de http:// www.racsa.co.cr/comunicados/ poblacion internet/index.html

Rodríguez, J. (2007). Un legado histórico-social y arquitectónico en el cantón de Palmares. Reflexiones. 86 (1), 141-150.

Romano, V. (2003). Educación ciudadana y medios de comunicación. Revista de Educación. (Núm. Extraordinario), 391-401.

Stake, R. (1999). Investigación con estudio de casos. Madrid, España: Ediciones Morata.

Villasuso, J.M. (2005). Interrogantes sobre la educación costarricense. En Marcos Arroyo y Juan Manuel Villasuso (Eds.), Dimensiones de la educación en Costa Rica. San José: Fundación Friedrich Ebert.

Zamora, C.M. (2000). El valle de los Palmares: historia de Palmares de Alajuela. San José: Ministerio de Cultura, Juventud y Deportes. 
